\title{
Homotypic Versus Heterotypic Continuity of Anxiety Symptoms in Young Adolescents: Evidence for Distinctions Between DSM-IV Subtypes
}

\author{
Robert F. Ferdinand · Gwen Dieleman · Johan Ormel • \\ Frank C. Verhulst
}

Published online: 17 January 2007

(C) Springer Science+Business Media, LLC 2007

\begin{abstract}
Objective: to investigate homotypic and heterotypic longitudinal patterns of symptoms of separation anxiety disorder (SAD), generalized anxiety disorder (GAD), social phobia (SoPh), panic disorder (PD), and obsessive compulsive disorder (OCD) in young adolescents from the Dutch general population.

Method: 2,067 individuals (51.4\% girls) from a Dutch community sample, who were assessed for the first time when they were aged 10 to 12 years, were followed up across a period of two years. At both assessments, anxiety symptoms were assessed with the RCADS, a self-report questionnaire.

Results: Regression analyses indicated that homotypic continuity was relatively high for SAD, GAD, and $\mathrm{SoPh}$ symptoms, and for PD in girls.

Conclusions: In many studies, anxiety disorders are treated as one group of disorders, and some widely used assessment instruments, such as the Child Behavior Checklist, do not even contain scales that tap different anxiety dimensions. In the present study, evidence for homotypic continuity was found, especially for symptoms of separation, social, and generalized anxiety, and for symptoms of panic disorder in girls, underscoring the usefulness of making distinctions between different anxiety constructs.
\end{abstract}

R. F. Ferdinand $(\bowtie) \cdot$ G. Dieleman · F. C. Verhulst Department of Child and Adolescent Psychiatry, Erasmus Medical Center, Rotterdam/Sophia Children's Hospital, Dr. Molewaterplein 60, P.O. Box 2060, 3000 CB Rotterdam, The Netherlands

e-mail: r.f.ferdinand@erasmusmc.nl

R. F. Ferdinand · J. Ormel

Department of Social Psychiatry, University of Groningen, Groningen, The Netherlands
Keywords Adolescence $\cdot$ Anxiety · Taxonomy

\section{Introduction}

Anxiety disorders are among the most prevalent psychiatric disorders in children and adolescents (Costello et al., 1996; Essau et al., 2000; Verhulst et al., 1997), are persistent, and are associated with impaired functioning (Canino et al., 2004; Ferdinand \& Verhulst, 1995; Ferdinand et al., 1995; McGee \& Stanton, 1990; Pine et al., 1998; Verhulst et al., 1997). The high degree of comorbidity amongst anxiety disorders in children and adolescents seems to point in the direction of one taxonomic construct, instead of a number of separate disorders. High comorbidity rates have been reported by many authors (Essau et al., 2000; Masi et al., 1999; Newman et al., 1996; Verduin \& Kendall, 2003). Evidence for a higher order factor that explains the presence of different types of anxiety has been found in children (Nauta et al., 2004) and adults (Hettema et al., 2005; Krueger, 1999; Vollebergh et al., 2001). Negative affectivity (NA) (Chorpita, 2002; Clark, 2005; Clark \& Watson, 1991; Lonigan et al., 1999, 2003) may be one of the higher order factors that may explain the finding of heterotypic continuity. NA represents displeasurable engagement with the environment and a sense of high subjective distress (Lonigan et al., 2003), and is often considered as a temperament trait that is associated not only with anxiety, but with depression as well (Clark, 2005).

Using data collected at the first assessment wave of a study that was also used to conduct the research that is being described in the present manuscript, support was found for the presence of one single anxiety dimension, instead of a number of separate anxiety concepts (Ferdinand et al., 2006). The sample of this previous study consisted of 10- to 12-year-olds 
from the Dutch general population, who completed a selfreport questionnaire for anxiety symptoms. Based on item scores on this questionnaire, latent class analysis did not detect classes of individuals with, for instance, high scores on items tapping separation anxiety, and simultaneously low scores on items tapping panic or social anxiety. Instead, high scores on one anxiety dimension implicated high scores on the other anxiety dimensions as well. However, other studies found evidence for separate anxiety dimensions. By performing factor-analyses, several authors found that DSM-IV anxiety disorders, such as generalized anxiety disorder, separation anxiety disorder, social phobia, and panic disorder represent different problem dimensions in children and adolescents (Chorpita \& Daleiden, 2000; Muris et al., 2002; Spence, 1997).

Longitudinal studies can provide valuable information regarding taxonomic constructs. For instance, it was found that symptoms of social phobia in adolescents predicted similar symptoms in adulthood (Pine et al., 1998). The prediction of a disorder by the same disorder is called homotypic continuity. However, social phobia symptoms also predicted simple phobia in adulthood. The prediction of a disorder by another disorder is called heterotypic continuity (Costello et al., 2003). Several mechanisms may explain heterotypic continuity. Heterotypic continuity may occur by chance. In other words, disease A may disappear, and disease B may occur subsequently, as a coincidence. However, in that case, continuity would not be reflected in statistical significance. More likely reasons for heterotypic continuity would be that disease $\mathrm{A}$ would be the cause of disease B, or that disease A and $\mathrm{B}$ share a common vulnerability factor.

The aim of the present study was to investigate homotypic and heterotypic longitudinal patterns of symptoms of separation anxiety disorder, generalized anxiety disorder, social phobia, panic disorder, and obsessive compulsive disorder in young adolescents from the Dutch general population. For this purpose, individuals from a community sample, who were assessed for the first time when they were aged 10 to 12 years, were followed up across a period of two years. At both assessments, anxiety symptoms were assessed with a self-report questionnaire. Given mixed results of previous studies, we did not formulate specific hypotheses regarding the level of homotypic or heterotypic continuity of different types of anxiety.

\section{Methods}

Sample and procedure

The TRacking Adolescents' Individual Lives Survey (TRAILS) is a prospective cohort study of Dutch early adolescents aged 10-12 years, who are followed biennially. The present study used data from the first (2001-2002) and second (2003-2004) assessment wave. The TRAILS target sample consisted of young adolescents from five municipalities in the North of the Netherlands, including both urban and rural areas. More details about the sample selection have been published elsewhere (de Winter et al., 2005).

Of all subjects who were approached at wave $1(N=3,145), 6.7 \%$ were excluded. The exclusion criteria were (1) adolescent incapable to participate because of mental retardation or a serious physical illness or handicap and (2) Dutch-speaking parent or parent surrogate not available, and not feasible to administer a part of the measurements in parent's own language. Of the remaining 2,935 young adolescents, $24 \%$ did not want to cooperate, and $76.0 \%$ cooperated with the study at wave $1(N=2,230$, mean age 11.09 years, $S D .55$, with $50.8 \%$ girls). Most frequent reasons for non-response were 'not interested' (33.8\%), participation in other research or unfavorable experiences with research $(15.4 \%)$, too much of a burden on the child (12.2\%), lack of time (10.3\%), concerns about privacy and confidentiality $(8.0 \%)$, and the child's refusal to participate because friend(s) did not participate $(4.0 \%)$. In 34 cases $(1.2 \%)$ we failed to contact anyone of the household (de Winter et al., 2005). Responders and non-responders did not differ with respect to the proportion of single parent families, or the prevalence of teacher-rated problem behavior. Furthermore, no differences between responders and non-responders were found regarding associations between socio-demographic variables and mental health outcomes (de Winter et al., 2005). To assess anxiety symptoms, the Revised Child Anxiety and Depression Scale (RCADS) (Chorpita \& Daleiden, 2000) was used at wave 1 , and also at wave 2 . For 20 cases, RCADS data were not obtained at wave 1 because respondents were not present during the measurements that were conducted in the classrooms, and could not be reached afterwards. Hence, RCADS data of 2,210 pre-adolescents were available at wave 1 .

At the second assessment wave, following similar procedures as at wave 1, RCADS information was obtained from 2,067 individuals. This was $95.5 \%$ of those for whom wave 1 RCADS information had been collected (51.4\% girls). To examine possible selective attrition, a stepwise logistic regression analysis was performed with 'wave 2 RCADS information available' as a dependent variable, and wave 1 age, sex, and the wave 1 RCADS Total Anxiety score (that was constituted by summing scores on the five anxiety dimensions that were assessed with the RCADS in the present study, see below) as possible predictors. The RCADS Total Anxiety score and sex did not predict attrition. However, younger age predicted attrition significantly (odds ratio $=.17$, Wald $=93.1, p<.001$; Model chisquare $=109,551, \mathrm{df}=1, p<.001)$. Cox and Snell $R$-square of the regression model was .048, which indicated 
Table 1 RCADS items

\author{
$S A D$ \\ Fears being alone at home \\ Scared to sleep alone \\ Scared to sleep away from home \\ Fears being away from parents \\ Worried in bed at night \\ Trouble going to school \\ Afraid of being in crowded places
}

\section{$G A D$}

Worried something awful will happen to family

Worried bad things will happen to self

Worried something bad will happen to self

Thinks about death

Worried about things

Worried about what will happen

$O C D$

Can't get silly/bad thoughts out of head

Keeps checking

Has to think thoughts to stop bad events

Has to do things over and over again

Has to do things just right to stop bad events

Bothered by bad/silly thoughts or images
SoPh

Worried when does poorly at things

Worried when somebody angry

Worried will do badly at school

Worried about mistakes

Worried what others think

Scared to take a test

Worried might look foolish

Afraid to talk in front of class

Afraid to look foolish in front of people

$P D$

Suddenly trouble breathing without reason

When has a problem, feels shaky

Suddenly trembling, shaking without reason

Suddenly dizzy, faint without reason

When has a problem, stomach feels funny

When has a problem, heart beats really fast

Suddenly feeling scared without reason

Suddenly heart beats too fast without reason

Worried suddenly get scared without reason that the effect of age was small. Further, most importantly, the level of anxiety at the initial assessment did not influence cooperation at wave 2 .

\section{Measures}

The Revised Child Anxiety and Depression Scale (RCADS) (Chorpita \& Daleiden, 2000) is a revision of the Spence Children's Anxiety Scale (SCAS) (Spence, 1997). It is a self-report questionnaire with 47 items, that are scored on a 4 -point scale $(0=$ never, $1=$ sometimes, $2=$ often, 3 = always). The questionnaire covers six scales, corresponding with DSM-IV dimensions of anxiety disorders and depressive disorder. The following five scales were used for the present study: separation anxiety disorder (SAD), generalized anxiety disorder (GAD), social phobia (SoPh), panic disorder (PD), and obsessive compulsive disorder (OCD) (see Table 1). The scale major depressive disorder (MDD) was not used. The internal consistencies of the scales that were used were (respectively at wave 1/wave 2) .66/.59 for $\mathrm{SAD}, .80 / .72$ for GAD, .78/.88 for $\mathrm{SoPh}, .75 / .72$ for PD, and $.68 / .66$ for OCD. The factor structure-for all six scales together - that was originally based on data from 1,641 children and adolescents from a community sample from Hawaii (Chorpita \& Daleiden, 2000), was confirmed by confirmatory factor analysis in the TRAILS sample at wave 1 (fit indices of NNFI $=.96$, RMSEA $=.05$, and $\mathrm{SRMR}=.05$, indicating an adequate fit to the sample data) (Ferdinand et al., 2006). The association of RCADS dimensions of anxiety with corresponding DSM-IV anxiety disorders was supported by previous research (Nauta et al., 2004).

\section{Statistical analyses}

First, to obtain information regarding comorbidity between different types of anxiety problems in the study sample, correlations among wave 1 RCADS scale scores were computed for each sex. Then, Pearson correlations were computed between wave 1 and wave 2 RCADS scale scores, separately for each sex group. Correlations provide insight in the associations between measures. However, by just computing correlations, it can not be judged if continuity is homotypic or heterotypic. For instance, the magnitude of a correlation between wave $1 \mathrm{SAD}$ and wave $2 \mathrm{SoPh}$ scores depends on the correlation between wave 1 SAD and wave $1 \mathrm{SoPh}$ scores. The higher correlations between wave $1 \mathrm{SAD}$ and wave $1 \mathrm{SoPh}$ are, the higher the correlation between wave $1 \mathrm{SAD}$ and wave $2 \mathrm{SoPh}$ will be. In other words, if assessment of continuity would solely be based on correlations, comorbidity at wave 1 would artificially inflate estimations of the extent of heterotypic continuity between wave 1 and wave 2 .

To correct for the effects of wave 1 comorbidity rates, regression analyses were conducted. First, it was assessed 
which part of continuity in anxiety problems was typically homotypic. For this purpose, for scores on each of the five RCADS scales at wave 2, a set of regression analyses was conducted, with wave 2 RCADS SAD, GAD, SoPh, PD, and OCD scores as dependent variables. These analyses were conducted to investigate how much of the variance in a specific RCADS scale score at wave 2 was not accounted for by an overall elevation in different types of anxiety at wave 1 , but instead, specifically by its own counterpart at wave 1 . We will now describe the regression analyses that were conducted for wave 2 SAD. Those for GAD, SoPh, PD, and OCD were similar. In the first block of the analyses, wave 1 scores on GAD, SoPh, PD, and OCD were entered simultaneously as predictors. Then, in a second block, wave 1 scores on the SAD scales were added, to see how much of the variance in wave 2 scores was predicted specifically by wave 1 SAD scores, and not by scores on the other RCADS scales at wave 1 . This variance reflects specific homotypic continuity. In the third block, sex was added. In the fourth block, an interaction between sex and SAD was added. If this interaction was significant, analyses were conducted for girls and boys separately. For each next block, the variance that was accounted for by the variable in this block was computed $\left(R^{2}\right)$.

Second, it was assessed which part of continuity in anxiety problems was specifically heterotypic. For this purpose, for scores on each of the five RCADS scales at wave 2, a set of regression analyses was conducted, with wave 2 RCADS SAD, GAD, SoPh, PD, and OCD scores as dependent variables. These analyses were conducted to investigate how much of the variance in a specific RCADS scale score at wave 2 was not accounted for by its own counterpart at wave 1 , but instead, by the other wave 1 anxiety scale scores. We will now describe the regression analyses that were conducted for wave 2 SAD. Those for GAD, SoPh, PD, and OCD were similar. In the first block of the analyses, wave 1 SAD scores were entered as predictor. Then, in the second block, scores on wave $1 \mathrm{GAD}$, SoPh, PD, and OCD scales were added, to see how much of the variance in wave 2 scores was specifically predicted by other RCADS scales at wave 1 . This variance reflects specific heterotypic continuity. In the third block, sex was added.

To judge the magnitude of effects, Cohen's rules for effects sizes can be used (Cohen, 1988). According to Cohen, $R^{2}$ between $1.0 \%$ and $5.9 \%$ is small, between $5.9 \%$ to $13.8 \%$ medium, and above $13.8 \%$ large.

\section{Results}

Means and standard deviations at wave 1 were calculated for SAD (mean $=.375, S D=.356)$, GAD $($ mean $=.666, S D=.454)$, SoPh $($ mean $=.779, S D=.427)$, $\mathrm{PD}($ mean $=.428, S D=.363)$, and OCD $($ mean $=.597$,
Table 2 Correlations among wave 1 RCADS scale scores

\begin{tabular}{lllll}
\hline \multirow{5}{*}{ Wave 1 RCADS scale } & \multicolumn{5}{l}{ Wave 1 RCADS scale } \\
\cline { 2 - 5 } & GAD b/g & SoPh b/g & PD b/g & OCD b/g \\
\hline SAD & $.52 / .58$ & $.52 / .52$ & $.51 / .52$ & $.47 / .51$ \\
GAD & - & $.59 / .54$ & $.55 / .54$ & $.58 / .58$ \\
SoPh & & - & $.56 / .55$ & $.54 / .53$ \\
PD & & & - & $.61 / .61$ \\
OCD & & & & - \\
\hline
\end{tabular}

Note. Correlations are presented for boys (b), girls (g) separately.

$S D=.445)$. Means and standard deviations were also calculated for wave $2 \mathrm{SAD}$ (mean $=.236, S D=.291$ ), GAD $($ mean $=.485, S D=.427)$, SoPh $($ mean $=.684, S D=.465)$, $\mathrm{PD}(\mathrm{mean}=.301, S D=.321)$, and OCD $($ mean $=.339$, $S D=.348$ ). Means reflect mean item scores for each RCADS scale.

Correlations among wave 1 RCADS scale scores for each sex separately are presented in Table 2 . It is shown that all correlations were in a close range, almost similar across sexes, and generally above .50 which can be regarded as high (Cohen, 1988). For both sexes the highest correlations were found between PD and OCD ( $r=.61$ in boys and girls), and the lowest between SAD and OCD (boys $r=.47$; girls $r=.51)$.

Correlations between wave 1 and wave 2 RCADS scale scores can be found in Table 3. For instance, in boys, the correlation between wave 1 and wave 2 SAD scores was .30 , whereas the correlations between wave $1 \mathrm{GAD}, \mathrm{SoPh}$, and PD scores and wave 2 SAD scores were .27, .29, and .22 respectively. Hence, heterotypic correlations were almost as high as the homotypic correlation. In girls, a similar result was found for wave 2 SAD. Heterotypic correlations ranged between .27 and .29 , whereas the homotypic correlation was .38. Similar relatively small discrepancies between homotypic and heterotypic correlations were found for wave $2 \mathrm{GAD}$, SoPh, and PD scores.

The results of the regression analyses are presented in Tables 4 and 5. Analyses that were aimed at assessing specific homotypic continuity (Table 4 ) indicated that variances

Table 3 Correlations between wave 1 and wave 2 RCADS scale scores

\begin{tabular}{llllll}
\hline \multirow{2}{*}{$\begin{array}{l}\text { Wave 1 } \\
\text { RCADS scale }\end{array}$} & \multicolumn{5}{l}{ Wave 2 RCADS scale } \\
\cline { 2 - 6 } & SAD b/g & GAD b/g & SoPh b/g & PD b/g & OCD b/g \\
\hline SAD & $.30 / .38$ & $.23 / .32$ & $.35 / .29$ & $.28 / .27$ & $.21 / .25$ \\
GAD & $.27 / .27$ & $.34 / .38$ & $.29 / .28$ & $.22 / .25$ & $.23 / .27$ \\
SoPh & $.29 / .29$ & $.35 / .29$ & $.42 / .41$ & $.29 / .28$ & $.25 / .25$ \\
PD & $.22 / .29$ & $.25 / .31$ & $.24 / .27$ & $.32 / .42$ & $.25 / .29$ \\
OCD & $.23 / .30$ & $.25 / .32$ & $.28 / .27$ & $.27 / .32$ & $.31 / .37$ \\
\hline
\end{tabular}

Note. Correlations are presented for boys (b), girls (g) separately. 


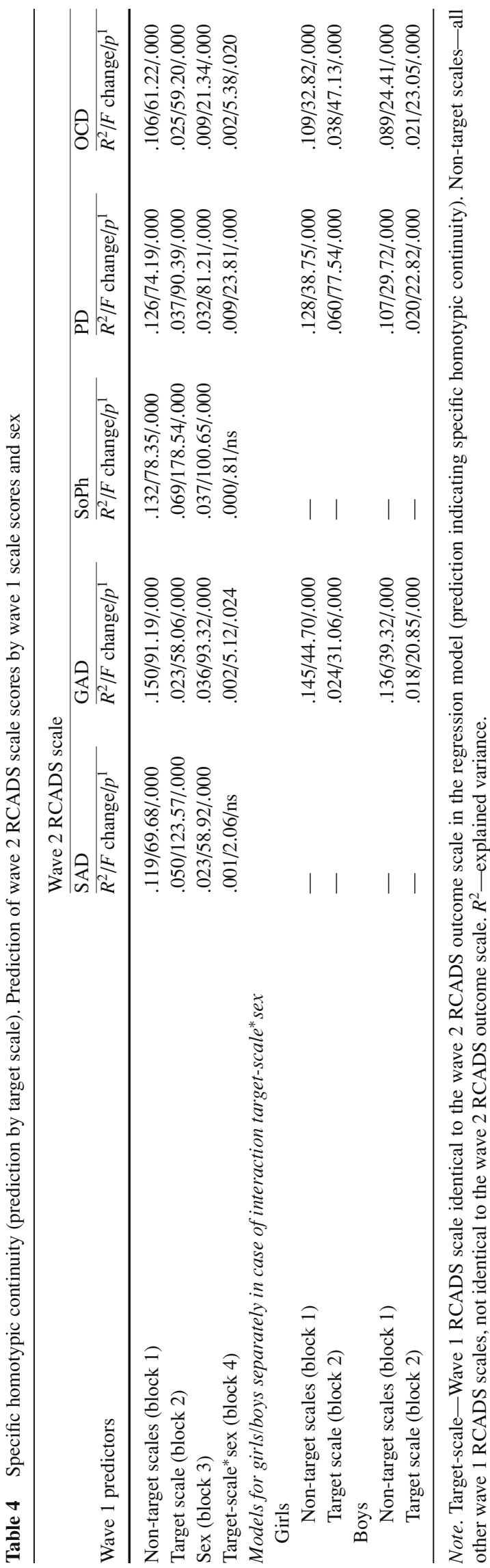

reflecting homotypic continuity were $2.3 \%$ for $\mathrm{SAD}, 3.6 \%$ for $\mathrm{GAD}, 3.7 \%$ for $\mathrm{SoPh}, 3.2 \%$ for PD, and .9\% for OCD. Analyses aimed as specifically investigating heterotypic continuity revealed variances of $2.0 \%$ for $\mathrm{SAD}, 4.2 \%$ for GAD, $1.3 \%$ for $\mathrm{SoPh}, 2.3 \%$ for $\mathrm{PD}$, and $2.7 \%$ for OCD (Table 5).

In analyses aimed at homotypic continuity, some anxiety* sex interactions were significant. A marked sex difference was found for PD. Table 4 shows that homotypic continuity was much stronger for girls (homotypic $R^{2}=6.0 \%$ ) than for boys (homotypic $R^{2}=2.0 \%$ ).

\section{Discussion}

The present study assessed homotypic and heterotypic continuity of symptoms of separation anxiety disorder, generalized anxiety disorder, social phobia, panic disorder, and obsessive compulsive disorder in individuals from a community sample, who were assessed for the first time when they were aged 10 to 12 years, and for the second time two years later. Variances reflecting homotypic continuity were roughly equal to those reflecting heterotypic continuity for SAD (2.3\% versus $2.0 \%)$ and GAD (3.6\% versus $4.2 \%)$. Variances reflecting homotypic continuity were larger than for heterotypic continuity for $\mathrm{SoPh}(3.7 \%$ versus $1.3 \%)$ and PD (3.2\% versus $2.3 \%)$, and smaller for homotypic than for heterotypic continuity for OCD (.9\% versus $2.7 \%$ ) (Tables 4 and 5). Applying cross-sectional research designs, previous studies found high comorbidity rates among different types of anxiety problems (Essau et al., 2000; Masi et al., 1999; Newman et al., 1996; Verduin \& Kendall, 2003). The present study extended the knowledge about the taxonomy of anxiety problems in young adolescents with longitudinal data. In accordance with previous cross-sectional work, SAD, GAD, $\mathrm{SoPh}, \mathrm{PD}$, and OCD symptoms appeared to be intertwined in a longitudinal fashion as well. However, considerable homotypic continuity was found as well.

Separation anxiety

For SAD, homotypic and heterotypic continuity were roughly equal. Previous studies indicated considerable comorbidity between SAD and the other anxiety problems, especially with SoPh (Compton et al., 2000). In the present study comorbidity rates at wave 1 were high as well, not only with $\mathrm{SoPh}$, but also with other types of anxiety. This suggests that SAD and the other anxiety problems may represent two sides of the same coin. However, homotypic continuity was as strong as heterotypic continuity, which supports the usefulness of SAD as a separate diagnostic construct. The distinction between SAD and other types of anxiety was further supported by another study, in which we conducted 
Table 5 Specific heterotypic continuity (prediction by non-target scales). Prediction of wave 2 RCADS scale scores by wave 1 scale scores and $\operatorname{sex}$

\begin{tabular}{|c|c|c|c|c|c|}
\hline \multirow[b]{3}{*}{ Wave 1 predictors } & \multicolumn{5}{|c|}{ Wave 2 RCADS scale } \\
\hline & SAD & GAD & SoPh & $\mathrm{PD}$ & OCD \\
\hline & $\overline{R^{2} / F \text { change } / p^{1}}$ & $\overline{R^{2} / F \text { change } / p^{1}}$ & $\overline{R^{2} / F \text { change } / p^{1}}$ & $\overline{R^{2} / F \text { change } / p^{1}}$ & $R^{2} / F$ change $/ p^{1}$ \\
\hline Target scales (block 1) & $.391 / 373.39 / .000$ & $.375 / 337.53 / .000$ & $.436 / 483.57 / .000$ & $.380 / 347.98 / .000$ & $.337 / 263.45 / .000$ \\
\hline Non-target scale (block 2) & $.020 / 83.77 / .000$ & $.042 / 86.59 / .000$ & $.013 / 103.79 / .000$ & $.023 / 80.00 / .000$ & $.027 / 82.20 / .000$ \\
\hline Sex (block 3) & $.027 / 81.60 / .000$ & $.041 / 90.95 / .000$ & $.040 / 107.45 / .000$ & $.028 / 82.80 / .000$ & $.012 / 55.90 / .000$ \\
\hline
\end{tabular}

Note. Target-scale-Wave 1 RCADS scale identical to the wave 2 RCADS outcome scale in the regression model. Non-target scales-all other wave 1 RCADS scales, not identical to the wave 2 RCADS outcome scale (prediction indicating specific heterotypic continuity). $R^{2}$-explained variance.

latent class analysis to assess the boundaries between SAD and $\mathrm{SoPh}$ in referred 8- to 11-year-olds (Ferdinand et al., 2006). Four different classes of individuals were detected; those with (1) low SAD and SoPh item scores on a self-report questionnaire, (2) high SAD and high SoPh item scores, (3) high SAD and low SoPh scores, and (4) low SAD and high $\mathrm{SoPh}$ scores. This also supported the idea that, despite high comorbidity rates, SAD may at least partially represent a separate phenomenon, that, for instance, may be subject to specific etiological influences that differ from influences that affect the course of other anxiety problems.

\section{Generalized anxiety}

For GAD, homotypic continuity was almost as strong as heterotypic continuity. This is - at least partly-consistent with a previous study (Pine et al., 2001) in adolescents from the general population indicating that separation anxiety disorder and social phobia in adolescents did not predict future generalized anxiety disorder. This suggested rather strong homotypic continuity of GAD. However, continuity of GAD was more heterotypic than in the Pine et al. study, because in our study, heterotypic continuity was also considerable. This contrast may be due to methodological issues such as differences in sample characteristics (Pine et al. investigated older adolescents), assessment procedures (Pine et al. applied standardized interviews instead of self-report questionnaires), or statistical approach (Pine et al. used categorical diagnostic samples whereas the present study used dimensional scale scores). Further, Pine et al. did not use a block design for their regression analyses, but included all predictors in a forward stepwise logistic regression analysis. So, in essence, they did not test if one predictor predicted future GAD, over and above the effect of other predictors.

\section{Social phobia}

Compared to heterotypic continuity, homotypic continuity of SoPh symptoms was relatively strong. Pine et al. (2001) found that $\mathrm{SoPh}$, but also GAD, in adolescence predicted future $\mathrm{SoPh}$, independently of other types of anxiety, whereas SAD did not. Remarkably, in their study, GAD was a bet- ter predictor of future $\mathrm{SoPh}$ than $\mathrm{SoPh}$ itself. The aforementioned methodological differences between the Pine et al. study versus the present study may explain differences between findings.

Panic

Homotypic and heterotypic continuity of PD did not differ much. Analyses for boys and girls separately (Table 4) indicated that homotypic continuity was higher in girls than in boys. Previous studies already indicated that panic disorder tends to have a chronic course, in children as well as in adults (Biederman et al., 1997; Bruce et al., 2005). The prevalence of full blown panic disorder, and even of panic attacks, in adolescents is very low (Essau et al., 1999). The present study nevertheless suggests that in girls, the disorder may already begin with a chronic homotypic course at a very young age which is in accordance with the higher prevalence in females versus males (Goodwin et al., 2005), and with studies that retrospectively investigated age at onset, and that often point to childhood or adolescence as a starting point (Sheehan et al., 1981).

Obsessive compulsive disorder

Comorbidity rates of OCD with other anxiety disorders are generally high (Heyman et al., 2001; Tukel et al., 2002), which was supported by the correlations among the wave 1 RCADS scale scores. The present study showed that heterotypic continuity of OCD symptoms was higher than homotypic continuity. To our knwoledge, previous studies that assessed homotypic continuity of OCD symptoms versus heterotypic continuity with other types of anxiety in young adolescents are not available, so we cannot compare our findings with previous work. Homotypic continuity was somewhat stronger in girls than in boys. This seems to suggest that, longitudinally, OCD symptoms in boys correlate differently with comorbid conditions than OCD symptoms in girls. Again, we were not able to find previous work on this topic. Future studies are needed to investigate if the differences between boys and girls we found can also be found in other samples, countries, and cultures. 
Practical implications

In many studies, anxiety disorders are treated as one group of disorders (Barrett et al., 2001; Lipman et al., 2001; MacMillan et al., 2001; Roza et al., 2003; Shortt et al., 2001), and, even, some widely used assessment instruments do not contain scales that tap different anxiety dimensions (Achenbach, 1991a, b). Previous studies found considerable associations between different types of anxiety symptoms, which suggested the presence of one higher order factor (Nauta et al., 2004). Several studies with adults also found evidence for a higher order factor that explained the presence of different types of anxiety (Hettema et al., 2005; Krueger, 1999; Vollebergh et al., 2001). Given the magnitude of heterotypic continuity in the present study, a higher order factor is likely to be present. However, the present study also showed that considerable homotypic continuity is present as well, occurring separately from a general propensity for high anxiety levels. This indicates that each type of anxiety problem may, at least partly, represent a distinct taxonomic construct. Homotypic continuity was found specifically for $\mathrm{SAD}, \mathrm{GAD}$, and $\mathrm{SoPh}$, and for PD in girls. This may indicate that $\mathrm{SAD}, \mathrm{GAD}, \mathrm{SoPh}$, and $\mathrm{PD}$ represent diagnostic constructs that are at least partially distinct. Hence, in clinical practice, instruments are needed that measure different anxiety dimensions separately. Instruments that just assess on single anxiety dimension may not be sufficient. Further, the distinctions between different anxiety constructs indicate that, despite the evidence that similar treatment methods are generally efficacious for different types of anxiety problems, each type of anxiety might require a slightly different treatment approach, and development of specific treatment modules.

\section{Limitations}

The sample consisted of young adolescents only. For older adolescents, different homotypic and heterotypic continuities could apply. Furthermore, questionnaires were used instead of clinical interviews; information about the presence or absence of DSM-IV (American Psychiatric Association, 1994) clinical diagnoses was not obtained. Even though RCADS symptom dimensions have proved to reflect corresponding DSM-IV anxiety disorders (Nauta et al., 2004), still, it may be the case that different results would have been obtained if DSM-IV diagnoses, that take account of the level of functional impairment, would have been used instead of RCADS scale scores.

Since different informants may provide different information, this study would have been more valuable if information regarding symptoms of different types of anxiety would also have been gathered from parents or teachers. Unfortunately, such information was not available.

\section{Conclusion}

In the present study's sample of young adolescents from the Dutch general population, evidence for homotypic continuity was found, especially for symptoms of separation, social, and generalized anxiety, and for symptoms of panic disorder in girls.

Acknowledgements This research is part of the TRacking Adolescents' Individual Lives Survey (TRAILS). We gratefully acknowledge the invaluable contribution of the staff members and fieldworkers during the preparation and execution of the data collection of TRAILS. Participating centers of TRAILS include various Departments of the University of Groningen, the Erasmus Medical Center of Rotterdam, the University of Nijmegen, the University of Utrecht, and the Trimbos Institute. TRAILS was financially supported by grants from the Dutch Organization for Scientific Research (GB-MW 940-38-011, GB-MAG 480-01-006, ZonMw 100-001-001 Geestkracht Program), the Dutch Ministry of Justice, the Sophia Foundation for Scientific Research (projects 301,393), and by the participating centers.

\section{References}

Achenbach, T. M. (1991a). Manual for the child behavior checklist/ 4-18 and 1991 profiles. Burlington, VT: Department of Psychiatry, University of Vermont.

Achenbach, T. M. (1991b). Manual for the youth self-report and 1991 profiles. Burlington, VT: University of Vermont Department of Psychiatry.

American Psychiatric Association. (1994). Diagnostic and statistical manual of mental disorders (4th ed.). Washington, DC: American Psychiatric Association.

Barrett, P. M., Duffy, A. L., Dadds, M. R., \& Rapee, R. M. (2001). Cognitive-behavioral treatment of anxiety disorders in children: Long-term (6-year) follow-up. Journal of Consulting Clinical Psychology, 69(1), 135-141.

Biederman, J., Faraone, S. V., Marrs, A., Moore, P., Garcia, J., Ablon, S., Mick, E., Gershon, J., \& Kearns, M. E. (1997). Panic disorder and agoraphobia in consecutively referred children and adolescents. Journal of the American Academy of Child \& Adolescent Psychiatry, 36, 214-223.

Bruce, S. E., Yonkers, K. A., Otto, M. W., Eisen, J. L., Weisberg, R. B., Pagano, M., Shea, M. T., \& Keller, M. B. (2005). Influence of psychiatric comorbidity on recovery and recurrence in generalized anxiety disorder, social phobia, and panic disorder: A 12-year prospective study. American Journal of Psychiatry, 162, 11791187.

Canino, G., Shrout, P. E., Rubio-Stipec, M., Bird, H. R., Bravo, M., Ramirez, R., Chavez, L., Alegria, M., Bauermeister, J. J., Hohmann, A., Ribera, J., Garcia, P., \& Martinez-Taboas, A. (2004). The DSM-IV rates of child and adolescent disorders in Puerto Rico: prevalence, correlates, service use, and the effects of impairment. Arch Gen Psychiatry, 61(1), 85-93.

Chorpita, B. F. (2002). The tripartite model and dimensions of anxiety and depression: An examination of structure in a large school sample. Journal of Abnormal Child Psychology, 30, 177190.

Chorpita, B. F., \& Daleiden, E. L. (2000). Properties of the Childhood Anxiety Sensitivity Index in children with anxiety disorders: Autonomic and nonautonomic factors. Behavior Therapy, 31(2), 327-349. 
Clark, L. A. (2005). Temperament as a unifying basis for personality and psychopathology. Journal of Abnormal Psychology, 114, 505521.

Clark, L. A., \& Watson, D. (1991). Tripartite model of anxiety and depression: Psychometric evidence and taxonomic implications. Journal of Abnormal Psychology, 100, 316-336.

Cohen, J. (1988). Power Analysis for the Behavioral Sciences (2nd ed.). Hillsdale, New Jersey: Lawrence Erlbaum Associates, Inc.

Compton, S. N., Nelson, A. H., \& March, J. S. (2000). Social phobia and separation anxiety symptoms in community and clinical samples of children and adolescents. Journal of the American Academy of Child and Adolescent Psychiatry, 39(8), 10401046.

Costello, E. J., Angold, A., Burns, B. J., Stangl, D. K., Tweed, D. L., Erkanli, A., \& Worthman, C. M. (1996). The great smoky mountains study of youth. Goals, design, methods, and the prevalence of DSM-III-R disorders. Archive of General Psychiatry, 53(12), $1129-1136$

Costello, E. J., Mustillo, S., Erkanli, A., Keeler, G., \& Angold, A. (2003). Prevalence and development of psychiatric disorders in childhood and adolescence. Archives of General Psychiatry, 60, 837-844.

de Winter, A. F., Oldehinkel, A. J., Veenstra, R., Brunnekreef, A., Verhulst, F. C., \& Ormel, J. (2005). Analysis of nonresponse bias in mental health determinants and outcomes in a large sample of preadolescents. European Journal of Epidemiology, 20, 173181.

Essau, C. A., Conradt, J., \& Petermann, F. (1999). Frequency of panic attacks and panic disorder in adolescents. Depress Anxiety, 9(1), 19-26.

Essau, C. A., Conradt, J., \& Petermann, F. (2000). Frequency, comorbidity, and psychosocial impairment of anxiety disorders in German adolescents. Journal of Anxiety Disorders, 14(3), 263279.

Feigon, S. A., Waldman, I. D., Levy, F., \& Hay, D. A. (2001). Genetic and environmental influences on separation anxiety disorder symptoms and their moderation by age and sex. Behavior Genetics, 31(5), 403-411.

Ferdinand, R. F., Bongers, I. L., Van Der Ende, J., van Gastel, W., Tick, N., Utens, E., \& Verhulst, F. C. (2006). Distinctions between separation anxiety and social anxiety in children and adolescents. Behavior Research and Therapy, 2006 Jan 11; [Epub ahead of print $]$.

Ferdinand, R. F., Van Lang, N. D. J., Ormel, J., \& Verhulst, F. C. (2006). No distinctions between different types of anxiety symptoms in pre-adolescents from the general population. Journal of Anxiety Disorders, 20, 207-221.

Ferdinand, R. F., \& Verhulst, F. C. (1995). Psychopathology from adolescence into young adulthood: An 8-year follow-up study. American Journal of Psychiatry, 152(11), 1586-1594.

Ferdinand, R. F., Verhulst, F. C., \& Wiznitzer, M. (1995). Continuity and change of self-reported problem behaviors from adolescence into young adulthood. Journal of the American Academy of Child and Adolescent Psychiatry, 34(5), 680-690.

Goodwin, R. D., Faravelli, C., Rosi, S., Cosci, F., Truglia, E., de Graaf, R., \& Wittchen, H. U. (2005). The epidemiology of panic disorder and agoraphobia in Europe. European Neuropsychopharmacology, 27 (in press).

Hettema, J. M., Prescott, C. A., Myers, J. M., Neale, M. C., \& Kendler, K. S. (2005). The structure of genetic and environmental risk factors for anxiety disorders in men and women. Archives of General Psychiatry, 62, 182-189.

Heyman, I., Fombonne, E., Simmons, H., Ford, T., Meltzer, H., \& Goodman, R. (2001). Prevalence of obsessive-compulsive disorder in the British nationwide survey of child mental health. British Journal of Psychiatry, 179, 324-329.

Krueger, R. F. (1999). The structure of common mental disorders. Archives of General Psychiatry, 56, 921-926.

Laurent, J., \& Ettelson, R. (2001). An examination of the tripartite model of anxiety and depression and its application to youth. Clinical Child and Family Psychology Review, 4, 209 230.

Lipman, E. L., MacMillan, H. L., \& Boyle, M. H. (2001). Childhood abuse and psychiatric disorders among single and married mothers. Am J Psychiatry, 158(1), 73-77.

Lonigan, C. J., Hooe, E. S., David, C. F., \& Kistner, J. A. (1999). Positive and negative affectivity in children: confirmatory factor analysis of a two-factor model and its relation to symptoms of anxiety and depression. Journal fo Consulting and Clinical Psychology, 67, 374-386.

Lonigan, C. J., Phillips, B. M., \& Hooe, E. S. (2003). Relations of positive and negative affectivity to anxiety and depression in children: Evidence from a latent variable longitudinal study. Journal of Consulting \& Clinical Psychology, 71, 465-481.

MacMillan, H. L., Fleming, J. E., Streiner, D. L., Lin, E., Boyle, M. H., Jamieson, E., Duku, E. K., Walsh, C. A., Wong, M. Y., \& Beardslee, W. R. (2001). Childhood abuse and lifetime psychopathology in a community sample. Am J Psychiatry, 158(11), $1878-1883$.

Masi, G., Mucci, M., Favilla, L., Romano, R., \& Poli, P. (1999). Symptomatology and comorbidity of generalized anxiety disorder in children and adolescents. Compr Psychiatry, 40(3), 210215.

McGee, R., \& Stanton, W. (1990). Parent reports of disability among 13-year olds with DSM-III disorders. Journal of Child Psychology and Psychiatry, 31(5), 793-801.

Muris, P., Schmidt, H., Engelbrecht, P., \& Perold, M. (2002). DSMIV-defined anxiety disorder symptoms in South African children. Journal of the American Academy of Child And Adolescent Psychiatry, 41(11), 1360-1368.

Nauta, M. H., Scholing, A., Rapee, R. M., Abbott, M., Spence, S. H., \& Waters, A. (2004). A parent-report measure of children's anxiety: psychometric properties and comparison with child-report in a clinic and normal sample. Behavior Research and Therapy, 42, 813-839.

Newman, D. L., Moffitt, T. E., Caspi, A., Magdol, L., Silva, P. A., \& Stanton, W. R. (1996). Psychiatric disorder in a birth cohort of young adults: prevalence, comorbidity, clinical significance, and new case incidence from ages 11 to 21. Journal of Consulting And Clinical Psychology, 64(3), 552-562.

Pine, D. S., Cohen, P., \& Brook, J. (2001). Adolescent fears as predictors of depression. Biological Psychiatry, 50(9), 721-724.

Pine, D. S., Cohen, P., Gurley, D., Brook, J. S., \& Ma, Y. (1998). The risk for early-adulthood anxiety and depressive disorders in adolescents with anxiety and depressive disorders. Archives of General Psychiatry, 55, 56-64.

Roza, S. J., Hofstra, M. B., Van Der Ende, J., \& Verhulst, F. C. (2003). Stable prediction of mood and anxiety disorders based on behavioral and emotional problems in childhood: a 14-year follow-up during childhood, adolescence, and young adulthood. American Journal of Psychiatry, 160(12), 2116-2121.

Sheehan, D. V., Sheehan, K. E., \& Minichiello, W. E. (1981). Age of onset of phobic disorders: A reevaluation. Comprehensive Psychiatry, 22, 544-553.

Shortt, A. L., Barrett, P. M., \& Fox, T. L. (2001). Evaluating the FRIENDS program: A cognitive-behavioral group treatment for anxious children and their parents. Journal of Clinical Child Psychology, 30, 525-535. 
Silberg, J. L., Rutter, M., \& Eaves, L. (2001). Genetic and environmental influences on the temporal association between earlier anxiety and later depression in girls: Erratum. Biological Psychiatry, 50(5), 393.

Spence, S. H. (1997). Structure of anxiety symptoms among children: a confirmatory factor-analytic study. Journal of Abnormal Psychology, 106(2), 280-297.

Tukel, R., Polat, A., Ozdemir, O., Aksut, D., \& Turksoy, N. R. (2002). Comorbid conditions in obsessive-compulsive disorder. Comprehensive Psychiatry, 43, 204-209
Verduin, T. L., \& Kendall, P. C. (2003). Differential occurrence of comorbidity within childhood anxiety disorders. Journal of Clinical Child Adolescence Psychology, 32(2), 290-295.

Verhulst, F. C., Van Der Ende, J., Ferdinand, R. F., \& Kasius, M. C. (1997). The prevalence of DSM-III-R diagnoses in a national sample of Dutch adolescents. Archives of General Psychiatry, 54(4), 329-336.

Vollebergh, W. A., Iedema, J., Bijl, R. V., de Graaf, R., Smit, F., \& Ormel, J. (2001). The structure and stability of common mental disorders: the NEMESIS study. Archives of General Psychiatry, $58,597-603$. 